PDES, SUBMANIFOLDS AND

AFFINE DIFFERENTIAL GEOMETRY

BANACH CENTER PUBLICATIONS, VOLUME 57

INSTITUTE OF MATHEMATICS

POLISH ACADEMY OF SCIENCES

WARSZAWA 2002

\title{
HYPERSURFACES WITH CONSTANT CURVATURE IN $\mathbb{R}^{n+1}$
}

\author{
J. A. GÁLVEZ and A. MARTÍNEZ \\ Departamento de Geometría y Topología \\ Facultad de Ciencias, Universidad de Granada \\ 18071 Granada, Spain \\ E-mail: jagalvez@ugr.es, amartine@ugr.es
}

\begin{abstract}
We give some optimal estimates of the height, curvature and volume of compact hypersurfaces in $\mathbb{R}^{n+1}$ with constant curvature bounding a planar closed $(n-1)$-submanifold.
\end{abstract}

1. Introduction. The compact hypersurfaces of constant positive curvature $K$ in $\mathbb{R}^{n+1}$ ( $K$-hypersurfaces) have been the principal objects of interaction between differential geometry theory of convex bodies and elliptic partial differential equations, specially those of Monge-Ampère type. Although many problems about their existence and uniqueness seem far from being understood, here we pose the problem of clarifying some properties about the geometry and topology of a $K$-hypersurface.

Since closed $K$-hypersurfaces are round spheres, the $K$-hypersurfaces of interest to us bound a connected submanifold of codimension 2 which lies in a hyperplane.

Let $S$ be a compact $n$-manifold with a nonempty connected boundary $\partial S$ and $x: S \rightarrow$ $\mathbb{R}^{n+1}$ be a $K$-hypersurface such that $\Gamma=x(\partial S)$ lies in a hyperplane $P$ of $\mathbb{R}^{n+1}$. First, we recall some elementary facts about $K$-hypersurfaces. Let $N$ and $\eta$ be unit normal vector fields along $S$ and $\partial S$ in $\mathbb{R}^{n+1}$ and $P$, respectively. Then, (up to sign) we have that

$$
\langle d N, d x\rangle=\langle N, \eta\rangle\langle d \eta, d x\rangle,
$$

along $\partial S$. This means that asymptotic directions on $\Gamma$ are also asymptotic on $x(S)$. We conclude that $\Gamma$ must be locally strictly convex and $P$ meets $x(S)$ transversally. Moreover, if $n \geq 3$, then the normal vector field $\eta: \partial S \rightarrow \mathbb{S}^{n-1}$ along $\partial S$ in $P$ is a global diffeomorphism and $\Gamma$ must be a hyperovaloid in $P$. Now, if $\Gamma$ is embedded, then by using for instance the results of Ghomi, see [6], we can find a connected hypersurface $M$ in $\mathbb{R}^{n+1}$ such that $x(S)+M$ is a hyperovaloid in $\mathbb{R}^{n+1}$. Consequently, there exists a

2000 Mathematics Subject Classification: Primary 53A05, 53A07.

Research partially supported by DGICYT Grant No. PB97-0785.

The paper is in final form and no version of it will be published elsewhere. 
convex body $U$ in $\mathbb{R}^{n+1}$ with $\partial U=x(S)+M$. Particularly, $x(S)$ must be embedded and it lies in one of the halfspaces determined by $P$.

Our goal in this paper is to prove some optimal estimates of the height, curvature and enclosed volume of hypersurfaces with positive constant curvature.

In $\S 2$ we prove two elliptic PDE's associated with the second fundamental form of the immersion which help us get height estimates for $K$-hypersurfaces (Theorems 1 and 2 ).

In $\S 3$ we derive a balancing formula which lets us obtain optimal curvature estimates of $K$-hypersurfaces bounding a connected $(n-1)$-hyperovaloid in $P$ (Theorem 3 ).

Finally in $\S 4$ we prove an estimation of the volume enclosed by a graph with constant curvature and boundary lying in a hyperplane.

2. Height estimates. In order to get an estimation of the maximum height at which a hypersurface with constant curvature can rise above a hyperplane, we calculate the laplacian with respect to the second fundamental form of the immersion and its Gauss map.

LEMma 1. Let $S$ be an orientable $n$-manifold and $x: S \rightarrow \mathbb{R}^{n+1}$ an immersion with Gauss map $N: S \rightarrow \mathbb{S}^{n}$ and a non-degenerate second fundamental form, $\sigma=-\langle d N, d x\rangle$. Then, the curvature of the immersion is constant if and only if

$$
\Delta^{\sigma} x=n N, \quad \Delta^{\sigma} N=-n H N,
$$

where $H$ is the mean curvature of the immersion and $\Delta^{\sigma}$ denotes the laplacian of the second fundamental form.

Proof. Let $\nabla$ and $\nabla^{\sigma}$ be the Levi-Civita connections of the usual metric of $\mathbb{R}^{n+1}$ and $\sigma$, respectively, and consider $\left\{E_{1}, \ldots, E_{n}\right\}$ an orthonormal moving frame in a neighbourhood of $p \in S$, parallel at $p$ for the metric $\sigma$, that is, $\sigma\left(E_{i}, E_{j}\right)=\varepsilon_{i} \delta_{i j}, \nabla_{E_{i}(p)}^{\sigma} E_{j}=0$, where $\varepsilon_{i}= \pm 1$ and $\delta_{i j}$ the Kronecker delta.

Using that $\left\langle\nabla_{E_{i}} N, E_{j}\right\rangle=-\sigma\left(E_{i}, E_{j}\right)=-\varepsilon_{i} \delta_{i j}$ we can calculate $\left\langle\Delta^{\sigma} N, E_{j}\right\rangle$ at $p$ :

$$
\begin{aligned}
\left\langle\Delta^{\sigma} N, E_{j}\right\rangle & =\sum_{i=1}^{n} \varepsilon_{i}\left\langle E_{i}\left(E_{i}(N)\right), E_{j}\right\rangle=\sum_{i=1}^{n} \varepsilon_{i}\left\langle\nabla_{E_{i}} \nabla_{E_{i}} N, E_{j}\right\rangle \\
& =\sum_{i=1}^{n} \varepsilon_{i}\left(E_{i}\left\langle\nabla_{E_{i}} N, E_{j}\right\rangle-\left\langle\nabla_{E_{i}} N, \nabla_{E_{i}} E_{j}\right\rangle\right) \\
& =\sum_{i=1}^{n} \varepsilon_{i}\left\langle-\nabla_{E_{i}} N, \nabla_{E_{i}} E_{j}\right\rangle .
\end{aligned}
$$

Moreover, if $G=\left(g_{k l}\right)=\left(\left\langle E_{k}, E_{l}\right\rangle\right)$ and $G^{-1}=\left(g^{l k}\right)$ is its inverse matrix, then

$$
-\nabla_{E_{i}} N=\sum_{l=1}^{n} \varepsilon_{i} g^{i l} E_{l} .
$$

Since the Lie bracket $\left[E_{i}, E_{j}\right](p)=0$, from $(2),(3)$ and Koszul formula, we obtain

$$
\left\langle\Delta^{\sigma} N, E_{j}\right\rangle=\sum_{i, l=1}^{n} g^{i l}\left\langle\nabla_{E_{i}} E_{j}, E_{l}\right\rangle=\frac{1}{2} \sum_{i, l=1}^{n} g^{i l}\left(E_{i}\left\langle E_{j}, E_{l}\right\rangle+E_{j}\left\langle E_{i}, E_{l}\right\rangle-E_{l}\left\langle E_{i}, E_{j}\right\rangle\right)
$$




$$
=\frac{1}{2} \sum_{i, l=1}^{n} g^{i l} E_{j}\left(g_{i l}\right)=\frac{1}{2} \operatorname{trace}\left(G^{-1} E_{j}(G)\right)=\frac{1}{2} E_{j}(\log (\operatorname{det}(G))),
$$

where det denotes the usual determinant.

As the curvature $K$ satisfies $|K| \operatorname{det}(G)=1$

$$
\left\langle\Delta^{\sigma} N, E_{j}\right\rangle=-\frac{1}{2} E_{j}(\log |K|) .
$$

Thus, it is clear that the tangent part of $\Delta^{\sigma} N$ vanishes if, and only if, $K$ is constant.

On the other hand, using (3)

$$
\begin{aligned}
\left\langle\Delta^{\sigma} N, N\right\rangle & =\sum_{i=1}^{n} \varepsilon_{i}\left\langle E_{i}\left(E_{i}(N)\right), N\right\rangle=\sum_{i=1}^{n} \varepsilon_{i}\left\langle\nabla_{E_{i}} \nabla_{E_{i}} N, N\right\rangle \\
& =\sum_{i=1}^{n} \varepsilon_{i}\left(E_{i}\left\langle\nabla_{E_{i}} N, N\right\rangle-\left\langle\nabla_{E_{i}} N, \nabla_{E_{i}} N\right\rangle\right) \\
& =\sum_{i, l=1}^{n} g^{i l}\left\langle E_{l}, \nabla_{E_{i}} N\right\rangle=-\sum_{i, l=1}^{n} g^{i l} \varepsilon_{i} \delta_{i l}=-\sum_{i=1}^{n} g^{i i} \varepsilon_{i} \\
& =-n H .
\end{aligned}
$$

In that way, from (4) and (5), $K$ is constant if and only if $\Delta^{\sigma} N=-n H N$ and we conclude the first assertion of the Lemma.

Now, the Codazzi equation gives

$$
\left\langle\nabla_{E_{i}} N, \nabla_{E_{j}} E_{k}\right\rangle=\left\langle\nabla_{E_{j}} N, \nabla_{E_{i}} E_{k}\right\rangle, \quad i, j, k=1, \ldots, n,
$$

and from (2), (3) and (4) we obtain:

$$
\begin{aligned}
\left\langle\Delta^{\sigma} x, E_{j}\right\rangle & =\sum_{i=1}^{n} \varepsilon_{i}\left\langle E_{i}\left(E_{i}(x)\right), E_{j}\right\rangle=\sum_{i=1}^{n} \varepsilon_{i}\left\langle\nabla_{E_{i}} \nabla_{E_{i}} x, E_{j}\right\rangle \\
& =\sum_{i=1}^{n} \varepsilon_{i}\left\langle\nabla_{E_{i}} E_{i}, E_{j}\right\rangle=\sum_{i, k=1}^{n} \varepsilon_{i} \varepsilon_{k} g_{j k}\left\langle\nabla_{E_{i}} E_{i},-\nabla_{E_{k}} N\right\rangle \\
& =\sum_{i, k=1}^{n} \varepsilon_{i} \varepsilon_{k} g_{j k}\left\langle\nabla_{E_{k}} E_{i},-\nabla_{E_{i}} N\right\rangle=\sum_{k=1}^{n} \varepsilon_{k} g_{j k}\left\langle\Delta^{\sigma} N, E_{k}\right\rangle \\
& =-\frac{1}{2} \sum_{k=1}^{n} \varepsilon_{k} g_{j k} E_{k}(\log |K|) .
\end{aligned}
$$

Moreover,

$$
\begin{aligned}
\left\langle\Delta^{\sigma} x, N\right\rangle & =\sum_{i=1}^{n} \varepsilon_{i}\left\langle E_{i}\left(E_{i}(x)\right), N\right\rangle=\sum_{i=1}^{n} \varepsilon_{i}\left\langle\nabla_{E_{i}} \nabla_{E_{i}} x, N\right\rangle \\
& =\sum_{i=1}^{n} \varepsilon_{i}\left\langle\nabla_{E_{i}} E_{i}, N\right\rangle=\sum_{i=1}^{n} \varepsilon_{i}\left\langle E_{i},-\nabla_{E_{i}} N\right\rangle=n .
\end{aligned}
$$

Since the matrix $\left(\varepsilon_{k} g_{j k}\right)$ has non-zero determinant, from (6) and (7), $K$ is constant if and only if $\Delta^{\sigma} x=n N$. 
As a consequence of the above Lemma we obtain (see [2], [3])

COROLlary 1. An orientable hypersurface in $\mathbb{R}^{n+1}$ with non-degenerate second fundamental form has constant curvature if and only if its Gauss map is harmonic for $\sigma$.

Lemma 1 and the Alexandrov reflection principle let us get estimates of the maximum height at which $K$-hypersurfaces can rise above a hyperplane. We also characterize the spherical caps as the unique graphs that reach those bounds.

Let $S$ be a compact $n$-manifold with a connected boundary $\partial S$ and consider a $K$ hypersurface $x: S \rightarrow \mathbb{R}^{n+1}$ such that

$$
\Gamma=x(\partial S) \subset P=\left\{p \in \mathbb{R}^{n+1}|\langle p, a\rangle=0,| a \mid=1\right\} .
$$

THEOREM 1. If $x$ is an embedding, then the maximum height at which $x(S)$ can rise above $P$ is $2 / \sqrt[n]{K}$.

Proof. Up to an isometry, we can assume that $a=(0, \cdots, 1)$ and $x(S)$ lies in $P^{+}=$ $\left\{p \in \mathbb{R}^{n+1} \mid\langle p, a\rangle \geq 0\right\}$. Since $x(S)$ is compact, there exists a point where every principal curvature has the same sign. Thus, $\sigma$ is definite.

By using, in a standard way, the Alexandrov reflection principle with respect to parallel hyperplanes to $P$ coming down from the highest point, $x(S)$ must be a graph, at least until the hyperplane is halfway down to $P$. Thus, it is sufficient to check that the bound $1 / \sqrt[n]{K}$ is satisfied if $x(S)$ is a graph.

We suppose $x(S)$ is a graph and choose the inner normal $N$. Then, $\sigma$ is positive definite and

$$
\Delta^{\sigma}(\sqrt[n]{K}\langle x, a\rangle+\langle N, a\rangle)=n(\sqrt[n]{K}-H)\langle N, a\rangle
$$

Since $H \geq \sqrt[n]{K}$, see [8], and $\langle N, a\rangle \leq 0$

$$
\Delta^{\sigma}(\sqrt[n]{K}\langle x, a\rangle+\langle N, a\rangle) \geq 0 \quad \text { on } S
$$

Now, bearing in mind that $\sqrt[n]{K}\langle x, a\rangle+\langle N, a\rangle \leq 0$ on the boundary, we have $\sqrt[n]{K}\langle x, a\rangle$ $+\langle N, a\rangle \leq 0$ on $S$ and the inequality follows.

THEOREM 2. If $x(S)$ is a graph on a compact domain in $P$ and the Euclidean gradient of height function, $\langle x, a\rangle$, is bounded along $\partial S$ (that is, there exists a real constant $m$ such that $|\nabla\langle x, a\rangle| \leq m \leq 1$ on $\partial S)$, then $\langle x, a\rangle \leq\left(1-\sqrt{1-m^{2}}\right) / \sqrt[n]{K}$.

Moreover, equality holds if and only if $x(S)$ is a spherical cap.

Proof. As before we can assume that $a=(0, \cdots, 1)$ and $x(S)$ lies in $P^{+}$. Consider the inner normal $N$, then $\langle N, a\rangle=-\sqrt{1-|\nabla\langle x, a\rangle|^{2}}$. Thus, on the boundary of $S$

$$
\sqrt[n]{K}\langle x, a\rangle+\langle N, a\rangle=\langle N, a\rangle \leq-\sqrt{1-m^{2}}
$$

and then, from (9), we have, $\sqrt[n]{K}\langle x, a\rangle+\langle N, a\rangle \leq-\sqrt{1-m^{2}}$ on $S$, that is,

$$
\langle x, a\rangle \leq \frac{-\langle N, a\rangle-\sqrt{1-m^{2}}}{\sqrt[n]{K}} \leq \frac{1-\sqrt{1-m^{2}}}{\sqrt[n]{K}} .
$$

Moreover, if equality holds, then there exists an interior point on the domain where $\sqrt[n]{K}\langle x, a\rangle+\langle N, a\rangle=-\sqrt{1-m^{2}}$. Using again (9) and the maximum principle the equality holds everywhere. Therefore, from (8), $H=\sqrt[n]{K}$ and $S$ is a spherical cap, see [8]. 
3. Curvature estimates. Let $S$ be a compact $n$-manifold with connected boundary $\partial S$ and $x: S \rightarrow \mathbb{R}^{n+1}$ an immersion such that the image of the boundary of $S$ lies in the hyperplane $P=\left\{p \in \mathbb{R}^{n+1}|\langle p, a\rangle=0| a \mid,=1\right\}$. Then, the number

$$
\bar{A}=\frac{1}{n} \int_{\partial S}\left\langle x \times d x \times{ }^{n-1)} \times d x, a\right\rangle
$$

is called the algebraic area of $x(\partial S)$. Moreover, the above number does not depend on the parametrization of the immersion and if $x(\partial S)$ is embedded then $|\bar{A}|$ is the volume enclosed by $x(\partial S)$ in $P$.

Recall from the Introduction that if $x$ is a $K$-hypersurface, then the curvature of $x_{\mid \partial S}$ in $P \equiv \mathbb{R}^{n}$ does not vanish at any point. Moreover, if $n \geq 3, x$ is an embedding.

Now, we obtain a necessary condition for a connected $(n-1)$-manifold lying in a hyperplane $P$ to be the boundary of a compact $K$-hypersurface.

Theorem 3. If $x: S \rightarrow \mathbb{R}^{n+1}$ is a $K$-hypersurface, then

$$
n K|\bar{A}| \leq \int_{\partial S} K_{\partial S} d A=\operatorname{vol}\left(\mathbb{S}^{n-1}\right) \operatorname{deg}(\eta),
$$

where $\eta$ is the Gauss map of $x: \partial S \rightarrow P$ and $K, K_{\partial S}>0$ denote the curvature of $S$ and $\partial S$ in $\mathbb{R}^{n+1}$ and $P$, respectively.

Moreover, equality holds if and only if $x(S)$ is a hemisphere.

Proof. Choose $N$ and $\eta$ such that $K$ and $K_{\partial S}$ are positive. It is clear that

$$
d N \times \stackrel{n}{.} . \times d N=K d x \times \stackrel{n}{.} . \times d x
$$

and using that $K$ is constant, we have

$$
\left.d\left(N \times d N \times{ }^{n-1} \cdots\right) \times d N\right)=d(K x \times d x \times \stackrel{n-1)}{\cdots} \times d x) .
$$

Then, from Stokes's theorem we obtain

$$
n K|\bar{A}|=K \int_{\partial S}\left\langle x \times d x \times{ }^{n-1} \cdots \times d x, a\right\rangle d A=\int_{\partial S}\left\langle N \times d N \times{ }^{n-1} \cdots \times d N, a\right\rangle d A,
$$

where $a$ is a unit normal vector to $P$ such that the above integral is non-negative.

On the other hand, there exists a real function $\theta$ such that $N=\cos \theta \eta+\sin \theta a$. Thus, $d N=d \theta(-\sin \theta \eta+\cos \theta a)+\cos \theta d \eta$ and

$$
\left.\left\langle N \times d N \times{ }^{n-1} \cdots\right) \times d N, a\right\rangle=(\cos \theta)^{n}\left\langle\eta \times d \eta \times{ }^{n-1} \cdots \times d \eta, a\right\rangle .
$$

Therefore,

$$
n K|\bar{A}|=\int_{\partial S}(\cos \theta)^{n} K_{\partial S} d A \leq \int_{\partial S} K_{\partial S} d A=\operatorname{vol}\left(\mathbb{S}^{n-1}\right) \operatorname{deg}(\eta) .
$$

Moreover, if equality holds then $\cos \theta=1$ along $\partial S$ and $N=\eta$, that is, $\langle N, a\rangle=0$ on $\partial S$. Hence, $x(S)$ meets $P$ orthogonally and $x(S)$ must be a graph on a convex domain in $P$ if $n \geq 3$.

In this way, for $n \geq 3$, using the Alexandrov reflection principle in any direction, $v$, perpendicular to $a, x(S)$ must be symmetric with respect to a hyperplane with normal vector $v$, see [11]. Therefore, $x(S)$ is a revolution hypersurface. 
Since equality holds $x(\partial S)$ must be a sphere of radius $1 / \sqrt[n]{K}$ and using again the Alexandrov reflection principle for graphs with the same boundary, $x(S)$ must be a hemisphere.

For $n=2$, since $\langle N, a\rangle=0, x(\partial S)$ is a line of curvature and its geodesic curvature vanishes identically. Thus, from the Gauss-Bonnet theorem

$$
2 \pi \chi(S)=\int_{S} K>0 .
$$

Therefore, the Euler characteristic of $S, \chi(S)$ is positive, that is, $\chi(S)=1$ and using Lemma 2 in [5], $x(S)$ is a hemisphere.

REMARK 1. Under the conditions of the above theorem:

1. If $S$ is a surface in $\mathbb{R}^{3}$, that is, $n=2$, then

$$
K|\bar{A}| \leq \pi|i(\partial S)|
$$

where $i(\partial S)$ is the rotation index of the curve $x(\partial S)$.

2. If $n \geq 3$,

$$
n K|\bar{A}| \leq \operatorname{vol}\left(\mathbb{S}^{n-1}\right)
$$

Now, we study compact graphs with non-zero curvature (not necessarily constant).

THEOREM 4. If $x(S)$ is a graph with non-zero positive curvature on a compact domain in the hyperplane $P$ and the Euclidean gradient of the height function is bounded by a real constant $m$ along $\partial S$ (that is, $|\nabla\langle x, a\rangle| \leq m \leq 1$ on $\partial S$ ), then

$$
n K_{0}|\bar{A}| \leq m^{n} \int_{\partial S} K_{\partial S} d A
$$

where $K, K_{\partial S}>0$ denote the curvature of $x(S)$ and $x(\partial S)$ in $\mathbb{R}^{n+1}$ and $P$, respectively, and $K_{0}$ is the minimum of $K$ on $x(S)$.

Moreover, equality holds if and only if $x(S)$ is a spherical cap.

Proof. We can consider $x(S) \subset P^{+}$. By taking the inner normals $N, \eta$ along $S$ and $\partial S$, respectively, and using the Stokes theorem

$$
\begin{aligned}
n K_{0}|\bar{A}| & \left.\left.=K_{0} \int_{\partial S}\left\langle x \times d x \times{ }^{n-1}\right) \times d x, a\right\rangle d A \leq \int_{\partial S} K\left\langle x \times d x \times{ }^{n-1}\right) \times d x, a\right\rangle d A \\
& =\int_{S} K d\left(\left\langle x \times d x \times{ }^{n-1)} \times d x, a\right\rangle\right) d A=\int_{S} d\left\langle N \times d N \times{ }^{n-1)} \times d N, a\right\rangle d A \\
& =\int_{\partial S}\left\langle N \times d N \times{ }^{n-1)} \times d N, a\right\rangle d A .
\end{aligned}
$$

Arguing as in the above theorem $N=\cos \theta \eta+\sin \theta a$ along $\partial S$ and

$$
n K_{0}|\bar{A}| \leq \int_{\partial S} \cos ^{n} \theta K_{\partial S} d A .
$$

Since $\sin \theta=\langle N, a\rangle=-\sqrt{1-|\nabla\langle x, a\rangle|^{2}}$ on $\partial S$ and $\cos \theta>0$ then $\cos \theta=|\nabla\langle x, a\rangle| \leq$ $m$ and the theorem follows.

If equality holds $K=K_{0}$ on $S$ and $|\nabla\langle x, a\rangle|=m$ on $\partial S$. Thus, using the Alexandrov reflection principle, as in the above theorem, $x(S)$ must be a spherical cap. 
4. Volume estimates. In this section, we give an estimation for the volume enclosed by a graph with constant curvature and planar boundary.

With the same notation as in $\S 3$ we have

THEOREM 5. If $x(S)$ is a K-hypersurface such that it is a graph on a compact domain in the hyperplane $P$ with bounded Euclidean gradient of the height function along $\partial S$ (that $i s$, there exists a real constant $m$ such that $|\nabla\langle x, a\rangle| \leq m \leq 1$ on $\partial S)$, then

(a) for $n=2$

$$
V \leq \frac{2-\sqrt{1-m^{2}}\left(2+m^{2}\right)}{3 \sqrt{K^{3}}} \pi
$$

(b) for $n \geq 3$

$$
n V \leq \frac{\operatorname{vol}\left(\mathbb{S}^{n-1}\right)}{\sqrt[n]{K^{n+1}}} \int_{\sqrt{1-m^{2}}}^{1} \sqrt{\left(1-t^{2}\right)^{n}} d t
$$

where $V$ is the volume enclosed by $S$ and $P$.

Moreover, equality holds if and only if $S$ is a spherical cap.

Proof. We can assume $x(S) \subset P^{+}$and $a=(0, \cdots, 1)$. Then,

$$
V=\int_{0}^{h}\left|\overline{A_{t}}\right| d t
$$

where $h$ is the maximum height of the graph above $P$ and $\overline{A_{t}}$ is the algebraic area of $B_{t}=S \cap\{\langle x, a\rangle=t\}$.

From (11),

$$
n K V=\int_{0}^{h}\left(\int_{B_{t}}|\nabla\langle x, a\rangle|^{n} K_{B_{t}} d A_{t}\right) d t
$$

curvature of $B_{t}$ in $\{\langle x, a\rangle=t\}$.

Then, from (10)

$$
1-|\nabla\langle x, a\rangle|^{2}=\langle N, a\rangle^{2} \geq\left(\sqrt{1-m^{2}}+\sqrt[n]{K}\langle x, a\rangle\right)^{2},
$$

and we have

$$
\begin{aligned}
n K V & \leq \int_{0}^{h}\left(\int_{B_{t}}\left(1-\left(\sqrt{1-m^{2}}+\sqrt[n]{K} t\right)^{2}\right)^{\frac{n}{2}} K_{B_{t}} d A\right) d t \\
& =\int_{0}^{h}\left(1-\left(\sqrt{1-m^{2}}+\sqrt[n]{K} t\right)^{2}\right)^{\frac{n}{2}}\left(\int_{B_{t}} K_{B_{t}} d A\right) d t .
\end{aligned}
$$

But, $\int_{B_{t}} K_{B_{t}} d A=\operatorname{vol}\left(\mathbb{S}^{n-1}\right)$ does not depend on $t$, consequently,

$$
n K V \leq \int_{0}^{h}\left(1-\left(\sqrt{1-m^{2}}+\sqrt[n]{K} t\right)^{2}\right)^{\frac{n}{2}} d t \operatorname{vol}\left(\mathbb{S}^{n-1}\right),
$$

and the theorem follows as in Theorem 4.

\section{References}

[1] L. A. Caffarelli, L. Nirenberg and J. Spruck, The Dirichlet problem for nonlinear secondorder elliptic equations I. Monge-Ampère equations, Comm. Pure Appl. Math. 37 (1984), 369-402. 
[2] J. Eells and L. Lemaire, A report on harmonic maps, Bull. London Math. Soc. 10 (1978), $1-68$.

[3] J. Eells and L. Lemaire, Another report on harmonic maps, Bull. London Math. Soc. 20 (1988), 385-524.

[4] J. A. Gálvez and A. Martínez, The Gauss map and second fundamental form of surfaces in $\mathbb{R}^{3}$, Geom. Dedicata 81 (2000), 181-192.

[5] J. A. Gálvez and A. Martínez, Estimates in surfaces with positive constant Gauss curvature, Proc. Amer. Math. Soc. 128 (2000), 3655-3660.

[6] M. Ghomi, Strictly convex submanifolds and hypersurfaces of positive curvature, J. Diff. Geometry 57 (2001), 239-271.

[7] B. Guan and J. Spruck, Boundary value problems on $\mathbb{S}^{n}$ for surfaces of constant Gauss curvature, Ann. of Math. 138 (1993), 601-624.

[8] G. Hardy, J. Littlewood and G. Pólya, Inequalities, Cambridge Univ. Press, 1989.

[9] D. Hoffman, H. Rosenberg and J. Spruck, Boundary value problems for surfaces of constant Gauss curvature, Comm. Pure Appl. Math. 45 (1992), 1051-1062.

[10] H. Rosenberg, Hypersurfaces of constant curvature in space forms, Bull. Sci. Math. 117 (1993), 211-239.

[11] M. Spivak, A Comprehensive Introduction to Differential Geometry, Publish or Perish, Inc., Berkeley, 1979. 